\title{
Different time course for age-related changes of behavior in a complex spatial cone-field discrimination task in Lewis rats
}

\author{
F. JOSEF VAN DER STAAY, ARJAN BLOKLAND, and WIJNAND RAAIJMAKERS \\ University of Limburg, Maastricht, The Netherlands
}

\begin{abstract}
Clear age-related impairments in learning and memory performance have consistently been reported for rodents in spatial discrimination tasks. The aim in the present study was to evaluate whether Lewis rats display age-associated changes in spatial working memory (WM) and reference memory (RM) performance and whether the changes in their performance on both memory components follow the same time course. Rats of five different ages of the Lewis strain were trained on a complex spatial discrimination task in a cone field. This task allows the simultaneous assessment of WM and RM. We observed that WM performance declined already between 4 and 12 months of age. RM performance, on the other hand, was better in the 18-, 24-, and 30-monthold animals than in the two youngest age groups. This was probably because the younger rats adopted the habit of extensively inspecting nonreinforced places. Correlation analysis of the data supported the view that WM and RM represent different aspects of spatial memory. The study corroborates the notion that distinct aspects of behavior decline differently with age.
\end{abstract}

Over the last few decades, a growing number of reports have been focused on the cognitive changes that accompany normal aging. In rats, age-related deficiencies in learning and memory performance are especially prominent in tasks that require the use of spatial information (Rapp, Rosenberg, \& Gallagher, 1987). Relative to young rats, senescent rats perform less well in complex spatial discrimination tasks such as the radial maze and circular platform (Barnes, Nadel, \& Honig, 1980), the Morris water maze (Gage, Dunnett, \& Björklund, 1984), and the holeboard (van der Staay, Raaijmakers, Sakkee, \& van Bezooijen, 1988; van der Staay, van Nies, \& Raaijmakers, 1990).

The distinction between working memory (WM) and reference memory (RM) has had a marked impact on theories about spatial discrimination learning (Olton, Becker, \& Handelmann, 1979). When only a fixed subset of places in an apparatus contains reinforcements, and when a rat is free to visit all places in that apparatus, these two memory components can be assessed simultaneously. The WM holds information about the places that have been visited during a trial. The RM holds information about the places that are baited; this information is trialindependent (Barnes, 1988).

Recently, we developed a cone field (see van der Staay, Krechting, Blokland, \& Raaijmakers, 1990) in order to study spatial discrimination learning. In the holeboard (Oades \& Isaacson, 1978), the predecessor of the cone

Requests for reprints should be mailed to W. G. M. Raaijmakers, Neuropsychology and Psychobiology, University of Limburg, P.O. Box 616, NL-6200 MD Maastricht, The Netherlands. field, no clear criteria exist to distinguish between accidental and directed orientation toward the target (hole). This ambiguity might lead to different operational definitions of a hole visit, which in turn might influence the learning curves for the WM and RM, as well as hamper the comparison of results from various studies (van Luijtelaar, van der Staay, \& Kerbusch, 1989). In the cone field, foodcups are placed in the tops of the cones. Every visit to the target (cone) is unambiguously operationally defined as a leaning response against the top of the cone (van der Staay, Raaijmakers, Lammers, \& Tonnaer, 1989). The visits are registered automatically. Clear age-related impairments of WM and RM performance have been observed in experiments done to compare aged female (33month-old) or aged male (29-month-old) Brown Norway rats with young (3-month-old) animals of the corresponding sex (van der Staay, Krechting, Blokland, \& Raaijmakers, 1990).

The cone-field task has been used to investigate the effects of bilateral fimbria transection on spatial WM and RM in Wistar rats (van der Staay et al., 1989). The transection produces cholinergic denervation, predominantly at the more ventral part of the hippocampus. Fimbrialesioned rats made about twice as many WM and RM errors as intact and sham-lesioned controls, even after extensive training. These results suggest that the cholinergic innervation of the ventral hippocampus plays a major role for the accurate performance in the cone-field task. This corroborates findings reported by others (summarized by Barnes, 1988) that postlesion acquisition of place versions of spatial discrimination tasks is impaired. Interestingly, no such impairments have been reported for cue versions of these tasks (Barnes, 1988). 
An experimental design including age groups covering the whole lifespan from adulthood to senescence is more adequate than an experiment with only two age groups, when one is studying age-related behavioral changes (Coleman, 1989; Coleman, Finch, \& Joseph, 1990; Ingram, 1985). We adopted such a multiple time point approach (Coleman et al., 1990) to compare the performance of 4-, 13-, 19-, and 30-month-old Brown Norway rats in a spatial holeboard discrimination task (van der Staay, van Nies, \& Raaijmakers, 1990). A clear agerelated decline in both WM and RM performance was found, an effect that was particularly pronounced between the ages of 19 and 25 months.

The present study was conducted to investigate, using a cone-field task, the age-related decline in spatial memory performance of Lewis rats of five different ages. We wondered whether the age differences observed in the holeboard study could be replicated in the cone field. We expected that both WM and RM performance would decline in older animals, but that the decline would not necessarily occur during the same period of the lifespan. Distinctive aspects of behavior may show different time courses with respect to age-related changes. For example, Gage and co-workers (Gage, Chen, Buzsaki, \& Armstrong, 1988) have presented experimental evidence for the independent decline of different cognitive and noncognitive abilities.

The complex spatial discrimination task in the cone field allows the simultaneous assessment of different aspects of a rat's behavior, as, for example, in detection of a preferred food search pattern and the measurement of spatial memory (discussed in van der Staay, Krechting, et al., 1990). We therefore considered the cone-field task to be especially suited for studying whether or not the different aspects of these behaviors have different time courses. In addition, we determined the interdependencies of WM and RM performance by correlation analysis, in order to test the notion put forward by Olton and Papas (1979) that the spatial WM and RM are independent. This analysis makes use of the broad range of performance expected because of the broad range of age groups involved in the study.

\section{METHOD}

\section{Animals}

We used 45 male inbred Lewis rats of five different ages: 4 months old $(n=10), 12$ months old $(n=8), 18$ months old $(n=10)$, 24 months old (range, 22-25 months; $n=8$ ), and 30 months old (range, 28-32 months; $n=9$ ). All animals except the 4- and 18month-old rats were retired breeders. The rats were healthy during and after the experiment; no motor impairments or cataracts were observed.

As far as we know, there are no exact figures about the mean lifespan of Lewis rats. Lindsay and coworkers (Lindsay, Nichols, \& Chaikoff, 1968) reported a survival rate of $26 \%$ at 24 months. We observed that the death rate of Lewis rats, which were kept in supply for experiments on aging in our animal house, started to increase from the age of 20 months, reaching a peak at about 28 months. Very few animals lived longer. The supplier of another line of Lewis rats (Charles River Wiga GmbH, West Germany) reports $50 \%$ survivability at $28-30$ months. Thus, animals from the two oldest groups might be considered as being senescent. The mean lifespan of Lewis rats is most likely shorter than that of Brown Norway rats (30-32 months; Burek, 1978), a strain that we have used in an aging study on spatial holeboard discrimination (van der Staay, van Nies, \& Raaijmakers, 1990).

All rats were housed individually in standard Makrolon cages on sawdust bedding in an air-conditioned room (ca. $20^{\circ} \mathrm{C}$ ). They were kept under a reversed light:dark cycle (lights on from 21:00 to $9: 00$ ).

\section{Apparatus}

The cone-field apparatus (see Figure 1) has been described in detail elsewhere (van der Staay et al., 1989). The construction of the cones, however, was modified in order to improve the measures taken to prevent the localization of the baits in the cones by odor cues (see Figure 1, lower right). All cones now contained a few inaccessible food pellets instead of just one. In addition, the height of the cones could now be adapted to the size of the rats, thereby making it necessary for rats to lean against the top of the cone in order to inspect the contents of a food cup (in the present study, the height of the cones was adjusted to $16 \mathrm{~cm}$ ).

The experimental room was illuminated by four red fluorescent tubes and three $100-\mathrm{W}$ bulbs, which were adjusted by a dimmer to give an intensity of about 50 lux on the floor of the apparatus. Extramaze cues consisted of two doors, a one-way screen and a window (both covered by black curtains), a sink, a table with computer and interface, and a radial maze. The experimenter sat in front of the apparatus. He was present and visible throughout all behavioral testing.

\section{Procedure}

Behavioral testing. There is a considerable increase in body weight with age in full-grown male Lewis rats: the 30-month-old rats are approximately $40 \%$ heavier than the 4 -month-old rats. This increase in weight from adulthood (4 months) to senescence (30 months) fits a linear regression equation:

body weight $($ grams) $=8.1 \cdot$ age (months) +398 .

A differential deprivation technique was applied in order to reduce motivational differences between age groups due to differences in body weight (Goodrick, 1968, 1980). Thus, the body weight of the rats was reduced to $85 \%$ ( 4 months), $80 \%$ (12 and 18 months), or $77.5 \%$ (24 and 30 months) of their free-feeding values within 1 week. This deprivation technique produces a more equivalent relative body weight loss across the age groups.

Familiarization with the cone field preceded formal training. The starting position was chosen randomly for each of these adaptation sessions. All cones contained one 45-mg food pellet (Bioserve Dustless Precision Pellets) during the adaptation sessions, and additional pellets were scattered on the floor of the apparatus. Familiarization was terminated as soon as at least 15 pellets were collected from the food cups of the cones within one session.

All rats were then trained in massed trials (Day 1,2 trials; Days 2 and 3, 3 trials; Day 4, 4 trials; Days 5-13, 6 trials), to a total of 60 trials. Within a series of daily trials, the starting positions were determined by random permutations of the numbers $1-4$. Each rat had its own order of starting positions.

Four cones of a fixed set contained one food pellet each. A trial was started by placing a rat in the startbox. The sliding door was then opened immediately. As soon as the rat had entered the cone field, the sliding door was closed. Whenever the rat touched the top of a cone, it closed an electrical circuit and activated a counter. This was scored automatically as a cone visit. Only contacts preceded by a visit to another cone were scored (exception: the first cone visit). Infrared photocells detected whether a rat poked its nose into 

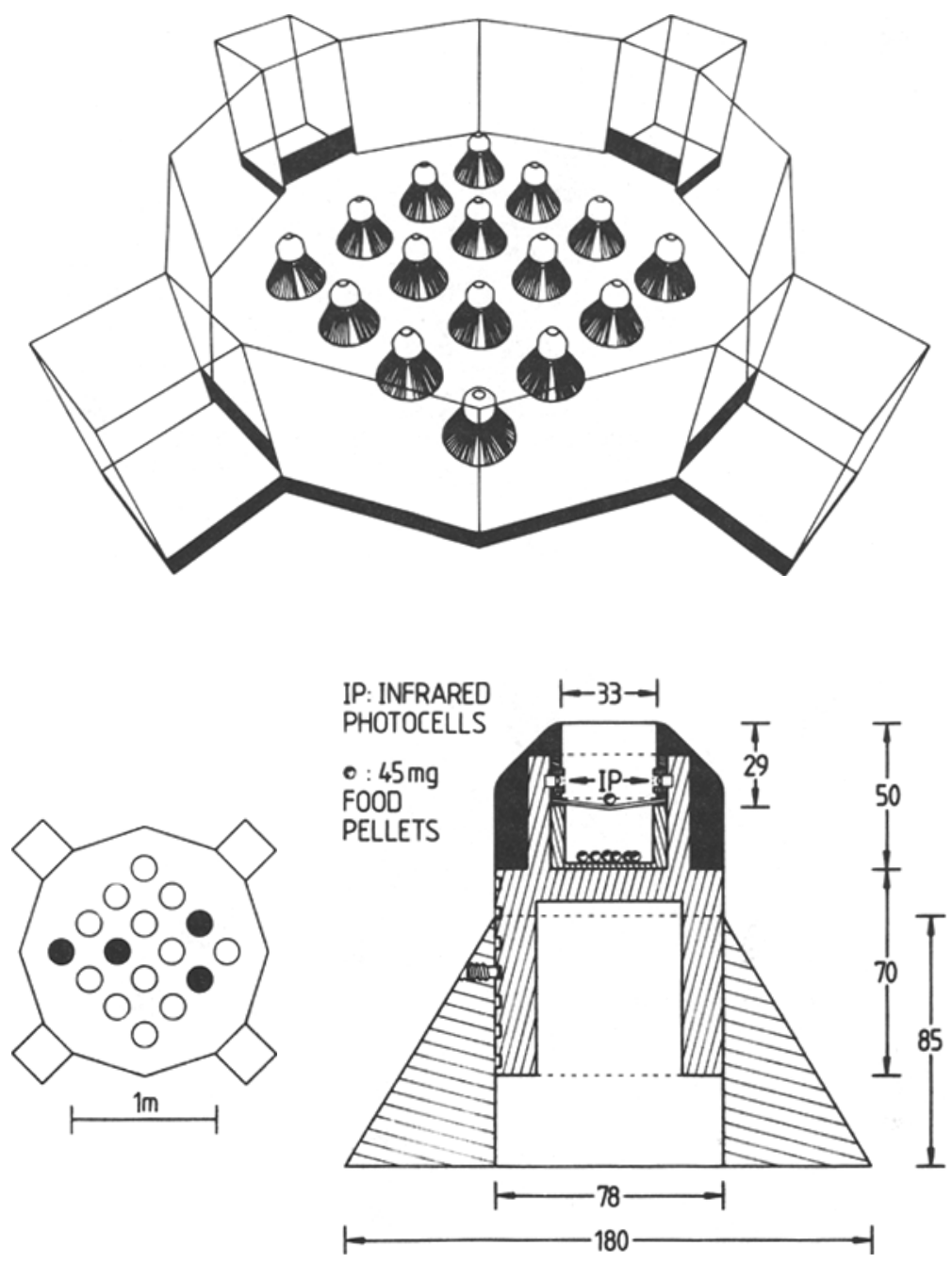

Figure 1. Upper panel: The cone-field apparatus. Lower left: Map of the cone field; filled circles are baited cones, open circles are unbaited cones. Lower right: Cross-section through a cone in the cone-field apparatus (measures are in millimeters). Cones were made of polyvinyl chloride, except for the upper part (drawn black), which was made of brass. A cone visit was automatically registered whenever a rat touched the upper part of the cone. The entire top of a cone can be dismantled easily to refill the reservoir of pellets that provide masking odor cues. The height of the cone could be adapted (lowest position, $124 \mathrm{~mm}$; highest position, $179 \mathrm{~mm}$ ).

a food cup. An IBM-compatible microcomputer collected the data and controlled the duration of the trials. A trial was terminated when the rat had found and consumed all four food pellets or when $7.5 \mathrm{~min}$ had elapsed, whichever event occurred first. The animal was put back into its home cage between trials. When the cone field had been cleaned with a damp sponge, and the four cones had been rebaited, the next trial was started. All testing was done between 9:00 and 13:00 (which corresponds to the first phase of the dark period).

Statistical analysis. Five measures of formal training were subjected to statistical analysis:

1. Working memory (WM: number of reinforced visits / number of visits and revisits to the baited set): This measure represents the percentage of visits to the baited set of cones that had been reinforced with a food pellet.

2. Reference memory (RM: total number of visits to the baited set / total number of visits): This measure represents the number of visits to the baited cones expressed as a percentage of all cone visits (baited and never baited).
3. General working memory (GWM: total number of cones visited / total number of visits): This measure represents the percentage of cone visits to the baited and never baited set that were visited at least once.

4. Choice correspondence of reinforced visits (CC): This measure reflects the variability of the pattern of visiting the baited set, but neglects all erroneous choices (cf. van der Staay et al., 1989).

5. Mean intervisit interval [IVI: time between the first and the last visit in a trial / (number of visits - 1)]: This variable provides a measure for the speed of visiting the cones.

Because there was a fairly high incidence of incomplete trials at the start of formal training, as indicated by the number of food rewards obtained (first trial block, mean number $\pm S E M$ of obtained food rewards: 4 months old, $3.5 \pm 0.19 ; 12$ months old, $3.0 \pm$ $0.19 ; 18$ months old, $3.5 \pm 0.08 ; 24$ months old, $3.1 \pm 0.19 ; 30$ months old, $3.1 \pm 0.22$ ), the analyses were performed over Trial Blocks 2-6 only. From the second trial block onwards, the rats rarely failed to collect all food rewards. Even a low incidence of incom- 
plete trials biases the error measures for WM, RM, and GWM. The ratio measures of WM, RM, and GWM are less biased by incomplete trials, and they are therefore presented here.

The means of blocks of 10 trials each were calculated for all variables. Age differences in the overall level of performance (general mean) and in the shape of the learning curves were analyzed by a one-factorial ANOVA on orthogonal trend components calculated over the successive trial blocks (Winer, 1971). Where appropriate, the results of ANOVAs on differences between ages for particular trial blocks are included. Duncan post hoc multiple range tests were performed in order to evaluate the age differences in more detail.

Product-moment correlation coefficients were calculated over all subjects for the measures WM and RM in order to evaluate their interrelationship. Again, analyses were performed for Trial Blocks 2-6 only.

\section{RESULTS}

Working memory. Averaged over all trial blocks (general mean), the WM performance (Figure 2A) was different for the age groups $[F(4,41)=9.26, p<.01]$. Post hoc analysis revealed that the 4-month-old animals performed better than the animals from the four older groups. All animals improved their WM performance in the course of training. This improvement was character-
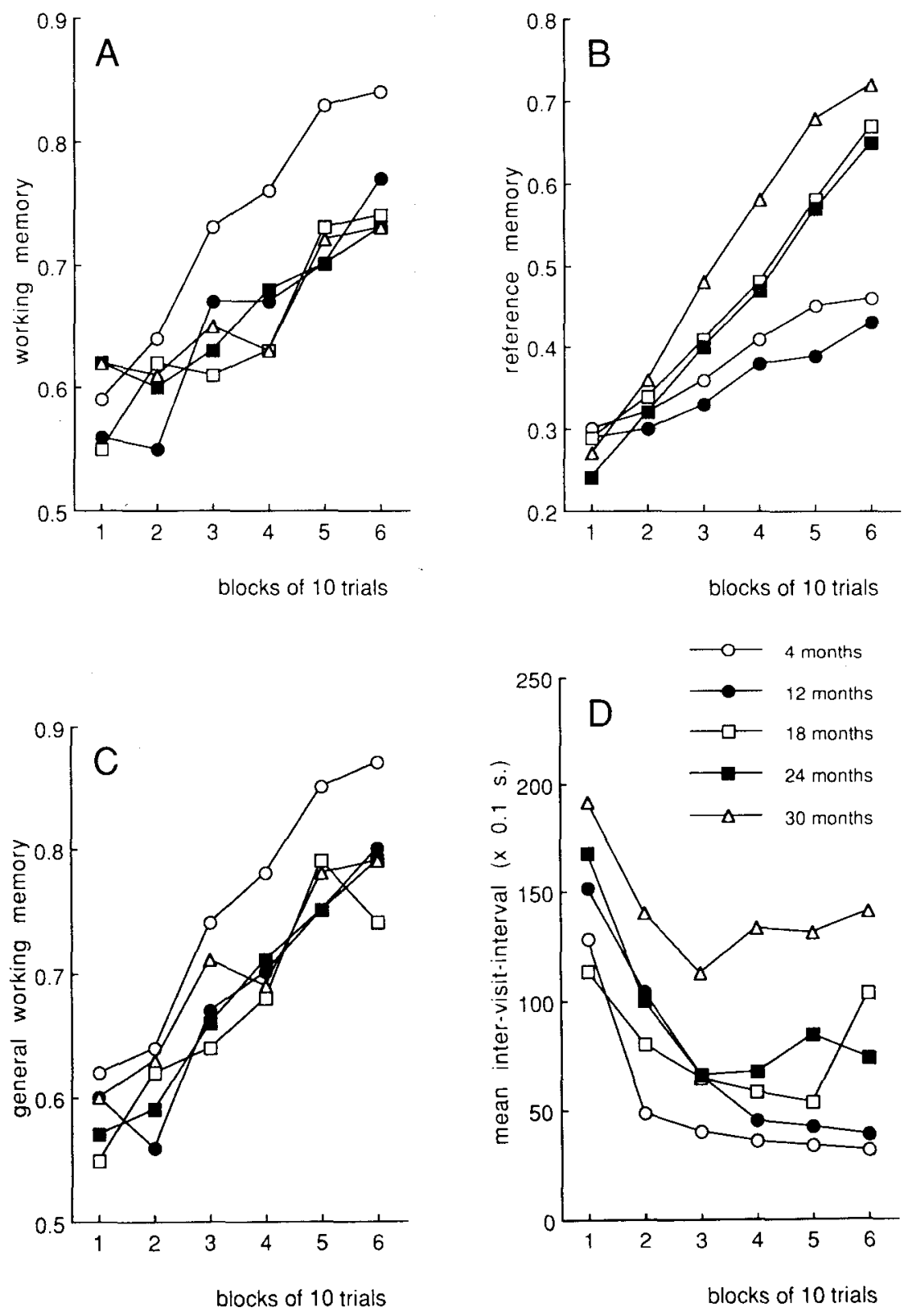

Figure 2. (A) working memory, (B) reference memory, (C) general working memory, and (D) intervisit interval $(\times 0.1 \mathrm{sec})$ of Lewis rats of five different ages in a spatial discrimination task in a cone-field apparatus. 
ized by a general linear trend $[F(1,41)=150.05, p<$ $.01]$, which explained $97 \%$ of the variation in the increase in WM performance. The age groups did not differ on the linear trend component $[F(4,41)=1.40$, n.s. $]$, indicating that the rate of improvement was similar for all ages.

Reference memory. In the first phase of formal training, no age differences were found for RM [Trial Block 2: $F(4,41)=1.77$, n.s.]. (See Figure 2B.) The general mean was different for the different age groups $[F(4,41)=$ $14.10, p<.01]$. Post hoc analysis of the general means revealed that the 30 -month-old rats performed best and that the 4- and 12-month-old rats performed worst. The intermediate performance of the 18- and 24-month-old animals differed from that of both the two youngest and oldest groups of rats. All age groups improved their performance in the course of training. This improvement was characterized by a general linear trend $[F(1,41)=387.23$, $p<.01$ ], which explained $99 \%$ of the variation. The age groups differed on the linear trend component $[F(4,41)=$ $14.94, p<.01]$. Post hoc analysis of the linear trend components revealed that the RM performance of the 4and 12-month-old animals improved more slowly than that of the three oldest groups.

General working memory. The picture for GWM (Figure $2 \mathrm{C}$ ) was very similar to that observed for WM. Averaged over trial blocks (general mean), the age groups showed different GWM performances $[F(4,41)=5.65$, $p<.01]$. Post hoc analysis confirmed that the performance of the 4-month-old animals was better than that of the rats in the other four age groups. The increase in GWM performance during training was characterized by a linear trend $[F(1,41)=349.09, p<.01]$, which predicted $97 \%$ of the variation. The age groups showed a parallel linear improvement in GWM performance $[F(4,41)=1.39$, n.s. $]$.

Choice correspondence of reinforced visits. There were no age differences in $\mathrm{CC}$ in any trial block. The CC fluctuated around the chance level. It is conceivable that the rats developed a fixed food-search pattern per start position. However, the $\mathrm{CC}$ still fluctuated around the chance level when this measure was analyzed separately for the four startboxes.

Mean intervisit interval. When averaged over Trial Blocks 2-6, the IVI of age groups differed [general mean: $F(1,41)=6.53, p<.01$ ] (see Figure 2D). Post hoc analysis revealed that the 30 -month-old rats visited the cones more slowly than did the younger animals, the IVIs of which did not differ from each other. The IVI approached an asymptote rapidly, as indicated by the absence of general linear, or quadratic trend components $[F(1,41)=0.58$, n.s., and $F(1,41)=0.96$, n.s., respectively). It should be noted that Trial Block 1 was not considered in the analysis.

The correlation analyses revealed that WM performance was not related to RM performance in any trial block.
Product-moment correlation coefficients for Blocks 2-6, calculated over all animals $(N=45)$, were $0.15,0.20$, $-0.04,-0.08$, and -0.27 , respectively (all associated probabilities: $p>.10$ ).

\section{DISCUSSION}

The WM performance of the young rats was better than that of the older rats in the spatial cone-field task, as was expected; however, the RM performance of the young rats was worse than that of the old rats. Although the 4-monthold rats continued to visit the unbaited set of cones in the course of training more often than the older animals (poor RM performance) did, they made fewer revisits to the baited cones (better WM performance) and to the baited and never baited cones combined (better GWM performance). Neither the young nor the old rats developed a fixed pattern of visiting the baited cones. The correlation analysis supports the notion of Olton and Papas (1979) that WM and RM represent different aspects of spatial discrimination performance.

The better WM performance of the young rats corroborates results from holeboard studies (van der Staay et al., 1988; van der Staay, van Nies, \& Raaijmakers, 1990 ) and from earlier studies with the cone field (van der Staay, Krechting, et al., 1990). The decrease in WM performance occurred between 4 and 12 months in the present cone-field experiment. The finding that the RM performance of the 4- and 12-month-old rats was worse than that of the older rats contrasts with the normal pattern of age-related decreases in spatial discrimination learning (e.g., Barnes et al., 1980; Gallagher \& Pelleymounter, 1988; van der Staay et al., 1990).

Our study involved two age groups consisting of virgin rats (4 and 18 months) and three age groups consisting of retired breeders. The breeding history could act as an intervening variable affecting the results of comparisons between groups of rodents (see Ingram, Spangler, \& Vincent, 1983). We feel that it is not likely that the lack of breeding experience was responsible for the poor RM performance of the 4-month-old rats, because the performance of the 18-month-old rats was not affected. However, we cannot rule this possibility out.

Although the 4-month-old rats continued to visit the never baited cones, even after extensive training, they avoided revisits to both the baited and the never baited set of cones, as is indicated by their better working memory performance (WM and GWM). Thus, the exploration of 4-month-old rats was very efficient. We assume that the 4- and 12-month-old rats have a tendency to investigate or explore the test environment more extensively than the older rats do. It may be of ecological advantage to continue to inspect previously baited cones, since they could potentially provide food (all cones had been baited during adaptation sessions). Although the 12month-old rats had the same tendency to explore their en- 
vironment as the 4-month-old animals did, they made more revisits due to impairments of working memory (as indicated by both WM and GWM).

The poor RM performance of the 4-month-old rats did not affect their efficiency to obtain the food rewardswhen the time needed to collect the baits is considered. Despite the fact that they visited more cones, the 4-monthold rats obtained their rewards faster than did the older rats [mean duration of trials of the last trial block in seconds \pm SEM: 4 months old, $42 \pm 3.6 ; 12$ months old, $56 \pm 7.7 ; 18$ months old, $52 \pm 7.4 ; 24$ months old, 71 $\pm 10.2 ; 30$ months old, $114 \pm 9.0 ; F(4,40)=14.29$, $p<.01]$.

An analysis of the number of different cones visited at least once during one trial provides an indication of the extent of exploration. All age groups visited approximately 12 different cones (including those of the baited set) in the second trial block $[F(4,40)=1.95$, n.s. $]$. The number of different cones visited decreased linearly with learning [linear trend: $F(1,40)=449.89, p<.01]$. The age groups differed with respect to this trend $[F(4,40)=$ $9.98, p<.01]$. Post hoc analysis confirmed that the decrease was more pronounced in the 18-, 24-, and 30month-old animals. In the last trial block, the mean numbers $( \pm S E M)$ of different cones visited by the 4-, 12-, 18-, 24-, and 30-month-old animals were, in order, 9.70 $( \pm 0.32), 10.6( \pm 0.43), 6.84( \pm 0.41), 7.48( \pm 0.52)$, and $6.56( \pm 0.26)$. The two youngest ages visited nearly the same number of cones as at the start of training; on the average, they inspected about $60 \%$ of all cones during a trial.

None of the age groups developed a fixed pattern of visiting the baited cones; the choice correspondence of reinforced visits fluctuated around the chance level. It appears that the young rats visited the cones in a rather arbitrary order, thereby relying heavily on their WM to avoid making revisits. They could have used scanning strategies, but these strategies were highly variable between and within subjects and were not detected by analysis of the data.

Olton $(1977,1983)$ has extensively investigated whether rats use a strategy or algorithm to solve the radial maze task (e.g., through development of a fixed order of arm visits). He found that no such strategies were used; the order of arm visits was too variable to depend on a particular rule or strategy. Olton concluded that rats relied on their WM. The choice behavior we observed for Lewis rats in the cone field is comparable to that reported for rats in radial maze studies; a response strategy was not detected.

It could be argued that the cones were so close together that it would have been difficult to use spatial cues. The standard eight-arm radial maze has a central platform with a diameter of approximately $35 \mathrm{~cm}$ (see, e.g., Olton \& Samuelson, 1976). The entrances of the arms are thus maximally $35 \mathrm{~cm}$ apart from each other. Rats are capable of discriminating with high accuracy between choice alternatives (arms) in such close proximity. In the cone field, the minimum distance between choice alternatives (tops of the cones) is $26.7 \mathrm{~cm}$; the maximum distance is $113.3 \mathrm{~cm}$ (outer cones of diagonals) (cf. van der Staay, Krechting, et al., 1990, Figure 1). The distance between choice alternatives (cones) thus appears to be sufficiently large to allow the use of extramaze cues in spatial orientation within the cone field.

There is another reason why the cone-field task appears to be more complex than, for example, the radial maze. In the cone field, an animal must learn to distinguish baited cones from unbaited cones, irrespective of its own position in space. In contrast, the choice point in the radial maze is always the same (the central platform), whereas every position within the cone field may serve as choice point for the next cone visit (van der Staay et al., 1989).

Figure 2 shows that there were age-related differences between the measures WM, GWM, RM, and IVI. For the working memory measures, the 4-month-old animals performed better than the older animals. The decline in WM and GWM performance in the cone-field task could already be seen in Lewis rats at an age of 12 months. On the other hand, the age difference in RM performance $o c$ curred between the ages of 12 and 18 months. A third measure, IVI, showed that the 30 -month-old animals visited cones more slowly than did animals in the other age groups. This supports the notion of others (e.g., Gage et al., 1988; Markowska et al., 1989) that the decline in performance for various aspects of behavior is heterogeneous and that the aging process is not controlled by one common underlying mechanism.

Jucker and colleagues (Jucker, Oettinger, \& Bättig, 1988) proposed that different physiological substrates underlie WM and RM. This assumption was based on their finding that, in an automated tunnel maze, the WM and RM performance of senescent rats (30 months) was impaired, whereas the WM but not the RM performance of middle-aged rats (17 months) was impaired, compared to the WM and RM performance of adult (5 months) animals. The authors considered that the results provided evidence for the hypothesis that the different physiological correlates underlying WM and RM are affected differently by aging. Following the line of reasoning of Jucker et al. (1988), one could argue that the better RM performance of the oldest rats in the present study was caused by an improved functioning of the underlying neural substrate. No experimental evidence, however, is available to support this view.

Lowy et al. (1985) used a modified T-maze procedure to investigate the age-related changes in WM and RM in rats. The RM component consisted of a spatial stem discrimination. WM was assessed by discrete-trial alternation performance in two different mazes. The aged (22-24 months) rats showed an impairment in both WM and RM performance when compared with adult (8-9 months) animals. Clear neurochemical correlates of the age-related decrease in spatial discrimination performance were not found.

To summarize, our data are in accordance with the idea that WM and RM represent different aspects of spatial discrimination performance. The notion that various as- 
pects of behavior may change independently of each other during the lifespan is supported by the present experiment, where the different behavioral measures were assessed simultaneously in the same animal and in the same testing environment. The present results are, however, probably limited to rats of the Lewis strain, since, in contrast with Lewis rats, both WM and RM performance in the cone-field task is better in adult Brown Norway rats than in senescent Brown Norway rats (van der Staay, Krechting, et al., 1990). It is likely that our results on RM reflect an age-associated change in the prevailing habit or strategy used to solve the cone-field task, rather than an improvement in the RM performance of the oldest animals.

The interpretation of measures for spatial WM and RM memory might be compromised by the development of extensive exploration tendencies. Thus, it is not appropriate to consider differences between young and old rats as an indication of a decline in spatial memory performance if age-related differences in the food-search habit occur. In that case, the age differences could (at least partly) be caused by the adoption of different behavioral strategies by the different age groups. This possibility should be kept in mind when one is studying age-related impairments of learning and memory.

In conclusion, attention should be paid to alternative explanations of the behavior operationally defined as WM and RM. Our results are consistent with Olton's (1988) notion that the heterogeneity of cognitive strategies and behavioral habits complicates the interpretation of agerelated changes. Therefore, spatial discrimination tasks should allow a detailed analysis of rats' behavior in order to detect possible age-related changes in the strategies used to solve a task.

\section{REFERENCES}

BARNES, C. A. (1988). Spatial learning and memory processes: The search for their neurobiological mechanisms in the rat. Trends in Neurosciences, 11, 163-169.

Barnes, C. A., NADEL, L., \& HoNiG, W. K. (1980). Spatial memory deficit in senescent rats. Canadian Journal of Psychology, 34, 29-39.

BureK, J. D. (1978). Pathology of aging rats. West Palm Beach, FL: CRC Press.

Coleman, P. D. (1989). How old is old? Neurobiology of Aging, 10, 115.

Coleman, P. D., Finch, C., \& JosePh, J. (1990). The need for multiple time points in aging studies. Neurobiology of Aging, 11, 1-2.

GaGe, F. H., Chen, K. S., Buzsaki, G., \& Armstrong, D. (1988). Experimental approaches to age-related cognitive impairments. Neurobiology of Aging, 9, 645-655.

GAGe, F. H., DunnetT, S. B., \& Buörklund, A. (1984). Spatial learning and motor deficits in aged rats. Neurobiology of Aging, 5, 43-48.

Gallagher, M., \& Pelleymounter, M. A. (1988). Spatial learning deficits in old rats: A model for memory decline in the aged. Neurobiology of Aging, 9, 549-556.

Goodrick, C. L. (1968). Learning, retention, and extinction of a complex maze habit for mature-young and senescent Wistar albino rats. Journal of Gerontology, 27, 298-304.

Goodrick, C. L. (1980). Problem solving and age: A critique of ro- dent research. In R. L. Sprott (Ed.), Age, learning ability and intelligence (pp. 5-25). New York: Van Nostrand Reinhold.

INGRAM, D. K. (1985). Analysis of age-related impairments in learning and memory in rodent models. Annals of the New York Academy of Sciences, 444, 312-331.

INGRAM, D. K., SPANGLER, E. L., \& VINCENT, G. P. (1983). Behavioral comparison of aged virgin and retired breeder mice. Experimental Aging Research, 9, 111-113.

JuCKer, M., OetTinger, R., \& BÄTtig, K. (1988). Age-related changes in working and reference memory performance and locomotor activity in the Wistar rat. Behavioral \& Neural Biology, 50, 24-36.

Lindsay, S., Nichols, C. W., \& Chaikoff, I. L. (1968). Naturally occurring thyroid carcinoma in the rat. Similarities to human medullary carcinoma. Archives of Pathology, 86, 353-364.

Lowy, A. M., Ingram, D. K., Olton, D. S., Waller, S. B., ReyNOLDS, M. A., \& London, E. D. (1985). Discrimination learning requiring different memory components in rats: Age and neurochemical comparisons. Behavioral Neuroscience, 99, 638-651.

Markowska, A. L., Stone, W. S., Ingram, D. K., Reynolds, J., Gold, P. E., Contr, L. H., Pontecorvo, M. J., Wenk, G. L., \& OLTON, D. S. (1989). Individual differences in aging: Behavioral and neurobiological correlates. Neurobiology of Aging, 10, 31-43.

OADES, R. D., \& ISAACSON, R. L. (1978). The development of food search behavior by rats: The effects of hippocampal damage and haloperidol. Behavioral Biology, 24, 327-337.

Olton, D. S. (1977). Spatial memory. Scientific American, 236(6), 82-98.

OLton, D. S. (1983). Memory functions and the hippocampus. In W. Seifert (Ed.), Neurobiology of the hippocampus (pp. 335-373). London: Academic Press.

OLton, D. S. (1988). Aging and spatial cognition. Neurobiology of Aging, 9, 569-570.

Olton, D. S., Becker, J. T., \& Handelmann, G. E. (1979). Hippocampus, space, and memory. Behavioral Brain Sciences, 2 , 313-365.

Olton, D. S., \& PAPAs, B. C. (1979). Spatial memory and hippocampal function. Neuropsychologia, 17, 669-682.

Olton, D. S., \& SAMUELSON, R. J. (1976). Remembrance of places passed: Spatial memory in rats. Joumal of Experimental Psychology: Animal Behavior Processes, 2, 97-116.

Rapp, P. R., Rosenberg, R. A., \& Gallagher, M. (1987). An evaluation of spatial information processing in aged rats. Behavioral Neuroscience, 101, 3-12.

van der StaAy, F. J., Krechting, B., Blokland, A., \& RaAumakers, W. G. M. (1990). The cone field: A spatial discrimination task for the automatic and simultaneous assessment of working and reference memory in rats. Journal of Neuroscience Methods, 31, 13-22.

van der Staay, F. J., Raaumakers, W. G. M., Lammers, A. J. J. C., \& Tonnaer, J. A. D. M. (1989). Selective fimbria lesions impair acquisition of working and reference memory of rats in a complex discrimination task. Behavioural Brain Research, 32, 151-161.

van der Staay, F. J., Raajmakers, W. G. M., Sakkee, A. N., \& VAN Bezoounen, C. F. A. (1988). Spatial working and reference memory of adult and senescent rats after thiopental anaesthesia. Neuroscience Research Communications, 3, 55-61.

van der Staay, F. J., van Nies, J., Ranumakers, W. (1990). The effects of aging in rats on working and reference memory performance in a spatial holeboard discrimination task. Behavioral \& Neural Biology, 53, 356-370..

van LuijtelaAr, E. L. J. M., van der StaAy, F. J., \& Kerbusch, J. M. L. (1989). Spatial memory in rats: A cross validation study. Quarterly Journal of Experimental Psychology, 41B, 287-306.

WINER, B. J. (1971). Statistical principles in experimental design (2nd ed.). New York: McGraw-Hill.

(Manuscript received April 11, 1990; accepted for publication May 9, 1990.) 